\title{
The "Young men may die, but old men must die" concept reappraised: The case of subdural hematomas
}

Subdural hematoma (SDH) is a condition commonly encountered in the everyday practice. Epidemiology data support an increasing incidence and prevalence trend. Only in the United States (between 1998 and 2007), 720,297 patients were hospitalized for SDH. Among them, more than $70 \%$ were aged over 60 years. The advance in age comes with an increase in dementia and a frequent use of antiplatelet drugs and vitamin $\mathrm{K}$ antagonists, as well as a plethora of risk factors involved in SDH appearance. ${ }^{[1]}$ The financial burden on health systems is significant. During this decade, the annual hospitalization cost for patients with $\mathrm{SDH}$ reached 1.6 billion US dollars, while the average cost for each admission for traumatic SDH was estimated to be 47,315 US dollars. ${ }^{[1,2]}$ Thus, it seems logical to focus our research efforts on the population of older patients suffering from SDH. The central question raised is whether these patients get operated and, if so, what is the outcome?

During the last few years, many studies were conducted with a special emphasis on elderly patients harboring a $\mathrm{SDH} .{ }^{[-6]}$ Acute $\mathrm{SDH}(\mathrm{aSDH})$ are diagnosed roughly in one-third of the patients with severe traumatic brain injury, and they are associated with high mortality rates. Taussky et al., retrospectively analyzed 37 Swiss patients ( $54 \%$ women, $46 \%$ men) who underwent craniotomy and duroplasty for aSDH. ${ }^{[3]}$ The median age was 73 years. Thirty patients $(81 \%)$ had significant comorbidities and $43 \%$ of patients were treated by anticoagulation or thrombocyte-aggregation inhibitors. The median initial Glasgow coma scale score (GCS) was 8 , and $51 \%$ patients presented with pupillary abnormalities. Perioperative morbidity occurred in 12 of 37 patients (32\%), and 13 patients died in the postoperative period (35\%). Overall, the outcome according to the Glasgow outcome scale (GOS) was

\begin{tabular}{|l|l|}
\hline \multicolumn{2}{|c|}{ Access this article online } \\
\hline Quick Response Code: & Website: \\
\hline & www.ruralneuropractice.com \\
\cline { 2 - 2 } & \\
\hline & \\
\hline
\end{tabular}

favorable (GOS, 4 and 5) in 15 of 37 patients (41\%); severely disabled (GOS, 3$)$ in 8 of $37(22 \%)$, and unfavorable (GOS, 1 and 2) in 14 of 37 (38\%). The authors concluded that craniotomy for patients aged more than 65 years remains controversial and that surgical treatment is associated with significant postoperative morbidity, mortality, and adverse outcome. However, selected patients benefit from an intervention, with a good outcome in $41 \%$ of patients.

Hanif et al., evaluated 29 cases (16 males, 13 females, all over 70 years of age) with aSDH that required surgical evacuation. ${ }^{[4]}$ Seventeen Irish patients sustained severe head injury (GCS 3-8). Mortality rate was 50\%, and the overall outcome was poor (GOS 3-5) (74.1\%). There were no functional survivors among those with severe head injury. The researchers questioned the efficiency of surgical intervention to prevent the poor outcomes associated with increased age, especially in the cases of severe head injury.

It is easily understood that the prognosis is not favorable in old patients presenting with aSDH. What about chronic SDH (cSDH)? De Araújo Silva et al., treated surgically 125 Brazilian patients with a median age of 69 (male/female ratio: 102/23). ${ }^{[5]}$ History of trauma was present in $60.8 \%$ of the patients. The median GCS on admission was 14 . The median GOS at 6 months was 4 (range: 1-5). One hundred and three patients obtained a good outcome (GOS 4-5), representing 82.4\% of the patients. Mortality rate was $11.2 \%$ (14 patients). Patients presenting with an admission GCS score $\geq 9$ had a statistically significant better outcome $(P=0.0033)$. However, age was not statistically associated with the outcome. The authors emphatically underlined the fact that older patients could actually benefit from surgical treatment and that they should be provided with that option.

Javadi et al., reported 40 patients with cSDH (28 males, 12 females) and a mean age of 67 years. ${ }^{[6]}$ From these, $7.5 \%$ presented with a GCS $<8$ on admission. Antiplatelets were identified as the risk factors in $20 \%$ of the patients. Good outcome (GOS 5) after 6 months was found in $60 \%$ of patients treated with drainage 
and in $50 \%$ of those with no drainage. The researchers concluded that the surgical technique did not seem to be the main variable improving the outcome of such patients. Other factors such as age, neurological status, and comorbidities seemed to have a more significant role on the surgical outcome.

Mulligan et al., presented their experience with 45 patients (33 men, 12 women) treated for aSDH, $\mathrm{cSDH}$, and mixed SDH. ${ }^{[7]}$ The mean age was 79.8 years (range: 70-94) and 28 patients were on anticoagulation therapy. The authors found no improvement in the functional outcome (3 patients died, $7 \%$ ). However, a significant neurological improvement was documented (Markwalder grading scale). Interestingly, improvement was observed in older patients with a worse preoperative neurological status in patients on anticoagulants and in patients with cSDH or mixed $\mathrm{SDH}$.

The literature findings are consistent with a better neurological and functional outcome in $\mathrm{cSDH}$ as compared to patients suffering from aSDH. It is true that, during the last years, the number of patients with $\mathrm{SDH}$ with a good discharge disposition has increased, while, at the same time, mortality has decreased. ${ }^{[1]}$ Physicians need to further explore the aging-associated neurobiological changes in order to better understand the observed outcome. ${ }^{[4]}$ It is recommended that the method of intervention should be determined by individualized decision making for each patient, taking into account the individual underlying risk factors. ${ }^{[5,6]}$ However, older patients should at least be considered for a surgical intervention (if not provided one). Advanced age should not be seen as an absolute contraindication for an operation in this subpopulation. As with young men, old men may die, but this does not mean that they must die...

Georgios K. Matis, Olga I. Chrysou, Theodossios A. Birbilis

Department of Neurosurgery, Medical School, Democritus University of Thrace, Alexandroupolis, Greece

Address for correspondence: Dr. Georgios K. Matis, 10 Plattenstrasse, $\mathrm{CH}-8032$, Zurich, Switzerland. E-mail: georgios.matis@uzh.ch

\section{References}

1. Frontera JA, Egorova N, Moskowitz AJ. National trend in prevalence, cost, and discharge disposition after subdural hematoma from 1998-2007. Crit Care Med 2011;39:1619-25.

2. Kalanithi P, Schubert RD, Lad SP, Harris OA, Boakye M. Hospital costs, incidence, and inhospital mortality rates of traumatic subdural hematoma in the United States. J Neurosurg 2011;115:1013-8.

3. Taussky P, Hidalgo ET, Landolt H, Fandino J. Age and salvageability: Analysis of outcome of patients older than 65 years undergoing craniotomy for acute traumatic subdural hematoma. World Neurosurg 2012;78:306-11.

4. Hanif S, Abodunde O, Ali Z, Pidgeon C. Age related outcome in acute subdural haematoma following traumatic head injury. Ir Med J 2009;102:255-7.

5. de Araújo Silva DO, Matis GK, Costa LF, Kitamura MA, de Carvalho Junior EV, de Moura Silva M, et al. Chronic subdural hematomas and the elderly: Surgical results from a series of 125 cases: Old "horses" are not to be shot! Surg Neurol Int 2012;3:150.

6. Javadi A, Amirjamshidi A, Aran S, Hosseini SH. A randomized controlled trial comparing the outcome of burr-hole irrigation with and without drainage in the treatment of chronic subdural hematoma: A preliminary report. World Neurosurg 2011;75:731-6.

7. Mulligan P, Raore B, Liu S, Olson JJ. Neurological and functional outcomes of subdural hematoma evacuation in patients over 70 years of age. J Neurosci Rural Pract 2013.;4:250-6

How to cite this article: Matis GK, Chrysou OI, Birbilis TA. The "Young men may die, but old men must die" concept reappraised: The case of subdural hematomas. J Neurosci Rural Pract 2013;4:245-6.

Source of Support: Nil. Conflict of Interest: None declared.

Announcement

\section{“QUICK RESPONSE CODE” LINK FOR FULL TEXT ARTICLES}

The journal issue has a unique new feature for reaching to the journal's website without typing a single letter. Each article on its first page has a "Quick Response Code". Using any mobile or other hand-held device with camera and GPRS/other internet source, one can reach to the full text of that particular article on the journal's website. Start a QR-code reading software (see list of free applications from http://tinyurl.com/yzlh2tc) and point the camera to the QR-code printed in the journal. It will automatically take you to the HTML full text of that article. One can also use a desktop or laptop with web camera for similar functionality. See http://tinyurl.com/2bw7fn3 or http://tinyurl.com/3ysr3me for the free applications. 